\title{
Ultrasonic phased array inspection of wire plus arc additive manufacture (WAAM) samples using conventional and total focusing method (TFM) imaging approaches
}

\begin{tabular}{|r|l|}
\hline Journal: & 57th Annual British Conference on Non-Destructive Testing \\
\hline Manuscript ID & NDT-0028-2018.R1 \\
\hline Topic: & Phased Arrays \\
\hline Date Submitted by the Author: & n/a \\
\hline Complete List of Authors: & $\begin{array}{l}\text { Javadi, Yashar; University of Strathclyde Department of Electronic and } \\
\text { Electrical Engineering, Centre for Ultrasonic Engineering (CUE) }\end{array}$ \\
\hline Keywords: & $\begin{array}{l}\text { Wire + Arc Additive Manufacture (WAAM), Ultrasonic Phased Array, Total } \\
\text { Focused Matrix (TFM), Artificial Defects, Full Matrix Capture (FMC) }\end{array}$ \\
\hline
\end{tabular}

\section{SCHOLARONE ${ }^{m}$ \\ Manuscripts}




\title{
Ultrasonic phased array inspection of wire plus arc additive manufacture (WAAM) samples using conventional and total focusing method (TFM) imaging approaches
}

\author{
Yashar Javadi $^{1 *}$, Charles N. MacLeod ${ }^{1}$, Stephen G. Pierce ${ }^{1}$, Anthony Gachagan ${ }^{1}$, William \\ Kerr $^{2}$, Jialuo Ding ${ }^{3}$, Stewart Williams ${ }^{3}$, Momchil Vasilev ${ }^{1}$, Riliang $\mathrm{Su}^{1}$, Carmelo Mineo ${ }^{1}$ and \\ Jerzy Dziewierz ${ }^{1}$ \\ ${ }^{1}$ Centre for Ultrasonic Engineering (CUE), Department of Electronic \& Electrical \\ Engineering, University of Strathclyde, Glasgow G1 1XQ, UK \\ ${ }^{2}$ Advanced Forming Research Centre (AFRC), University of Strathclyde, 85 Inchinnan \\ Drive, Renfrew PA4 9LJ, UK \\ ${ }^{3}$ Welding Engineering and Laser Processing Centre, School of Aerospace, Transport and \\ Manufacturing Building 46, Cranfield University, Cranfield, Bedfordshire MK43 0AL, UK \\ *Corresponding author's email: yashar.javadi@strath.ac.uk
}

\begin{abstract}
In this study, three aluminium samples produced by wire + arc additive manufacture (WAAM) are inspected using ultrasonic phased array technology. Artificial defects are machined using a centre drill, $\varnothing 3 \mathrm{~mm}$, and electro-discharge machining (EDM), $0.5-1 \mathrm{~mm}$, in a cylindrical through hole topology. The sample was first inspected by a single element wheel probe mounted on a KUKA robot in order to investigate the feasibility of using a conventional ultrasonic transducer approach. Unfortunately, the wheel probe was found to be unsuitable for scanning of the WAAM specimens and ultrasonic phased arrays were employed next. The setup included $5 \mathrm{MHz}$ and $10 \mathrm{MHz}$ arrays (128 elements) in direct contact with the sample surface using both conventional and total focusing method (TFM) imaging techniques. Using FIToolbox (Diagnostic Sonar, UK) as the controller, a phased array aperture of 32 elements was used to perform a focussed B-scan with a range of settings for the transmit focal depth. All the reflectors (including those located near the WAAM top surface) were successfully detected by a combination of conventional phased array and TFM, using a range of settings and setups including bottom surface inspection, application through a Plexiglas wedge and variation of the scanning frequency.
\end{abstract}

Keywords: Wire + Arc Additive Manufacture (WAAM); Ultrasonic Phased Array; Total Focusing Method (TFM); Artificial Defects; Full Matrix Capture (FMC). 


\section{Introduction}

Additive manufacture (AM), technologies that build 3D samples by adding layer-upon-layer of material, have become increasingly popular because they facilitate manufacturing of more cost-effective parts by reducing the material wastage and time to market, an increase in design freedom, weight saving, as well as manufacturing of complex but single-part geometries rather than assemblies made of many subcomponents [1]. The importance of wastage minimisation is highlighted by the aircraft designers who are forced to shift from aluminium to titanium (an expensive material to source and machine) because the aluminium is not electrochemically compatible with the carbon used in the increasingly popular carbon fibre components [2]. Therefore, it is critical to consider an alternative manufacturing process for large aircraft components (e.g., stiffened panels, wing ribs, etc.) rather than machining from billets or large forgings which lead to an undesirably high value, 10 or even 20, of buyto-fly (BTF) ratio - the weight ratio between the raw material used for a component and the weight of the component itself [3]. A suitable alternative is wire + arc additive manufacture (WAAM) which has been successfully employed for depositing large components $(>10 \mathrm{~kg}$ in titanium, aluminium, steel, etc.) with delivering high deposition rates, low material and equipment costs, and good structural integrity [3]. Using arc welding tools and wire as feedstock for additive manufacturing purposes, WAAM has shown low BTF ratios and low capital and feedstock costs. For example, Williams et al [3] showed a $2.5 \mathrm{~m}$ aluminium wing rib was manufactured by WAAM at $1.1 \mathrm{~kg} / \mathrm{hr}$ delivering a BTF ratio of 12 rather than machining from solid with a BTF ratio of 37 and then WAAM enabled material savings in excess of roughly $500 \mathrm{~kg}$ per part [3].

Application of ultrasonic array, a single transducer that contains the number of individually connected elements, for non-destructive evaluation has dramatically increased during the recent years due to a great potential to increase inspection quality and reduce inspection time [4]. The ultrasonic array advantages in comparison with traditional single element transducer methods are an increased flexibility, an ability to produce rapid visualisation, undertaking a range of different inspections from a single location, scanning with an index of the width of the array, synthetic aperture focusing and the ability to scan a beam over a range of angles and positions, without moving the transducer [4, 5]. These advantages along with developments such as three-dimensional volumetric imaging of components using twodimensional arrays [6], testing of components with complex geometries using high temperature arrays [7] and flexible arrays [8] to be employed in harsh environments 
particularly nuclear and aerospace industries as well as showing significant promise for NDE through the air coupled arrays $[9,10]$ have led to the rapid uptake of arrays by the engineering industry.

The ultrasonic array allows real-time images (B-scans) to be generated in three standard inspection techniques: plane B-scan; focused B-scan and sector B-scan [11]. These three Bscan approaches utilise a set of time-domain data (A-scans) and if this is captured from all combinations of transmitting and receiving elements, a full matrix capture (FMC) is achieved which can maximise the flexibility of array signal processing and extract as much information as possible from an array $[11,12]$. Total focusing method (TFM) is an imaging algorithm that uses data acquired in FMC mode for post-processing and then all elements in the array are employed to focus at every single point in the image [11].

Structural integrity assessment and non-destructive testing (NDT) of the WAAM specimens are required to be developed in parallel with the rapid industrialisation of the WAAM and AM processes. The necessity of standardisation in the field of AM is reflected in the joint collaboration between International Organization for Standardization (ISO) and ASTM, ISO/TC 261, in which NDT for AM parts, ISO/TC 261/JG 59, is supposed to be published by the end of 2019 [13]. Clark et al [14] reviewed NDT for AM parts while the particular focus of this study, the WAAM-NDT, was reviewed by Lopez et al [15]. Application of radiography and ultrasonic method on the WAAM components was also studied by Lopez et al [16]. They employed single element ultrasonic probe to detect the WAAM defects while the requirement of a good surface finish was reported as a disadvantage of this method [16].

In this study, the WAAM specimens were non-destructively tested by the ultrasonic phased array and using both conventional and TFM approaches. However, the necessity of using the phased array rather than the traditional single element ultrasonic method is firstly studied in an experimental setup using a KUKA robot and an ultrasonic wheel probe. The aperture and setting of the phased array system are also improved in order to detect as many artificial defects as possible particularly those located near the top surface of the WAAM samples.

\section{Sample and defect specifications}

Three aluminium samples, shown in Figure 1, were inspected ultrasonically in order to locate the artificial defects which were produced on the as-built samples, ALT1 and ALT2. The sample ALT3, on the other hand, was machined to flatten the WAAM top and side surfaces 
and then electro-chemical-machine (EDM) was employed to make the holes. The artificial defects and the sample specifications are also detailed in Table 1. There is a backing plate (aluminium) on which the first layer is deposited and then the WAAM sample is manufactured. Due to the machining of WAAM top surface in the ALT3, it was possible to place the ultrasonic transducers over either the top WAAM surface or the backing plate (referred to as bottom surface scanning in this study) while scanning of the ALT1 and ALT2 was only possible from the bottom surface because the top WAAM surface was not flat.

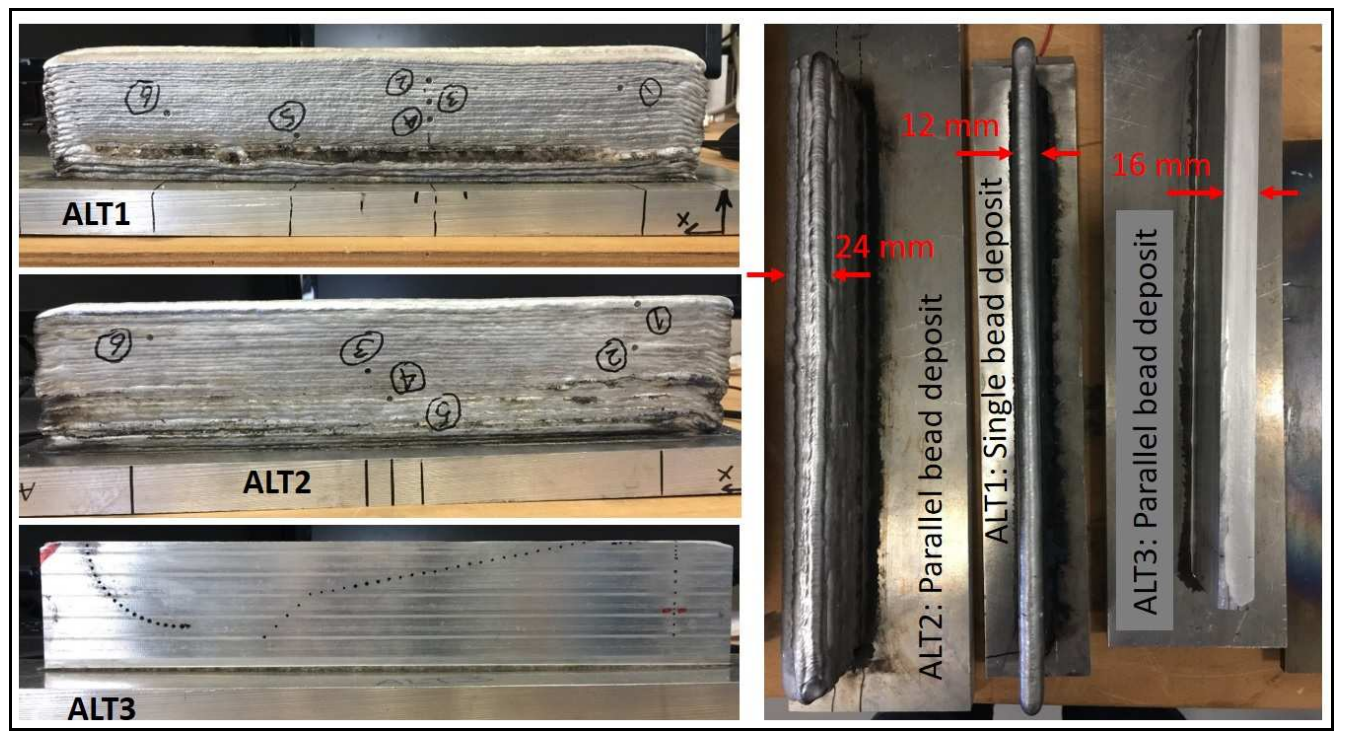

Figure 1. Three aluminium samples investigated in this study

Table 1. Sample and defects specifications

\begin{tabular}{|c|c|c|c|}
\hline $\begin{array}{c}\text { WAAM } \\
\text { sample }\end{array}$ & Material & WAAM sample specifications & Defect specifications \\
\hline ALT1 & Aluminium & $\begin{array}{c}\text { Parallel Bead Deposit (As built) }-20 \text { Layers }- \\
300 \mathrm{~mm} \text { long }\end{array}$ & 6 holes (Ø3 mm) by drill \\
\hline ALT2 & Aluminium & $\begin{array}{c}\text { Single Bead Deposit (As built) }-20 \text { Layers }- \\
300 \mathrm{~mm} \text { long }\end{array}$ & 6 holes (Ø3 mm) by drill \\
\hline ALT3 & Aluminium & $\begin{array}{c}\text { Parallel Bead Deposit (machined and EDM) }- \\
20 \text { Layers }-300 \mathrm{~mm} \text { long }\end{array}$ & $\begin{array}{c}60 \text { holes (ø0.5-1 mm) by } \\
\text { EDM }\end{array}$ \\
\hline
\end{tabular}

\section{Single element wheel probe}

One of the samples (ALT1) was firstly tested using a traditional single element ultrasonic transducer in order to study the feasibility of WAAM-NDE using the single element transducer. The setup included a wheel probe mounted on a KUKA robot, Figure 2, in order to provide an automated robotic NDE system which was a final goal of a larger project (RoboWAAM) supporting this sub-project work. 


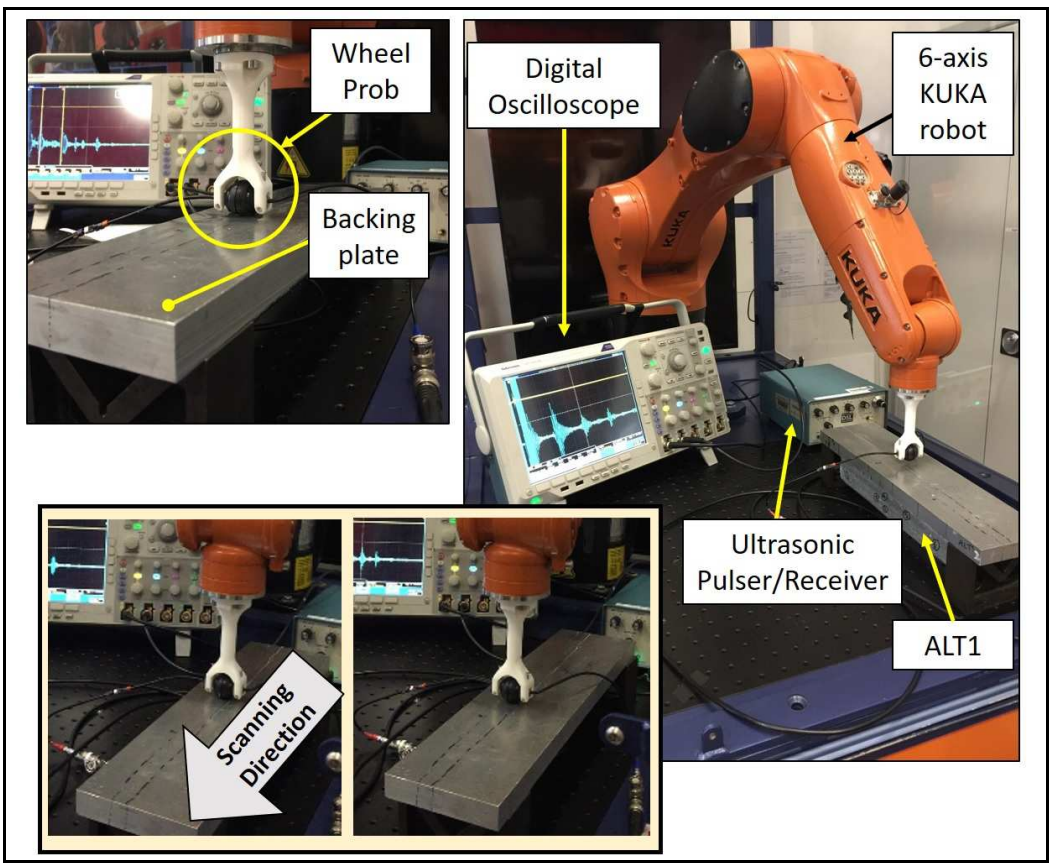

Figure 2. Inspection of the WAAM sample (ALT1) using a wheel probe (single element transducer)

The inspection results, see Figure 3, showed that even $3 \mathrm{~mm}$ hole is not detectable by the single element scanning of the WAAM sample. It might be possible to improve these scanning images if a flat, single-element probe (rather than the wheel probe) and/or a focused beam transducer is employed. However, the difficulty of locating the simulated defects using single element scanning in the multi-layer welding and WAAM specimens justifies application of the phased array system.

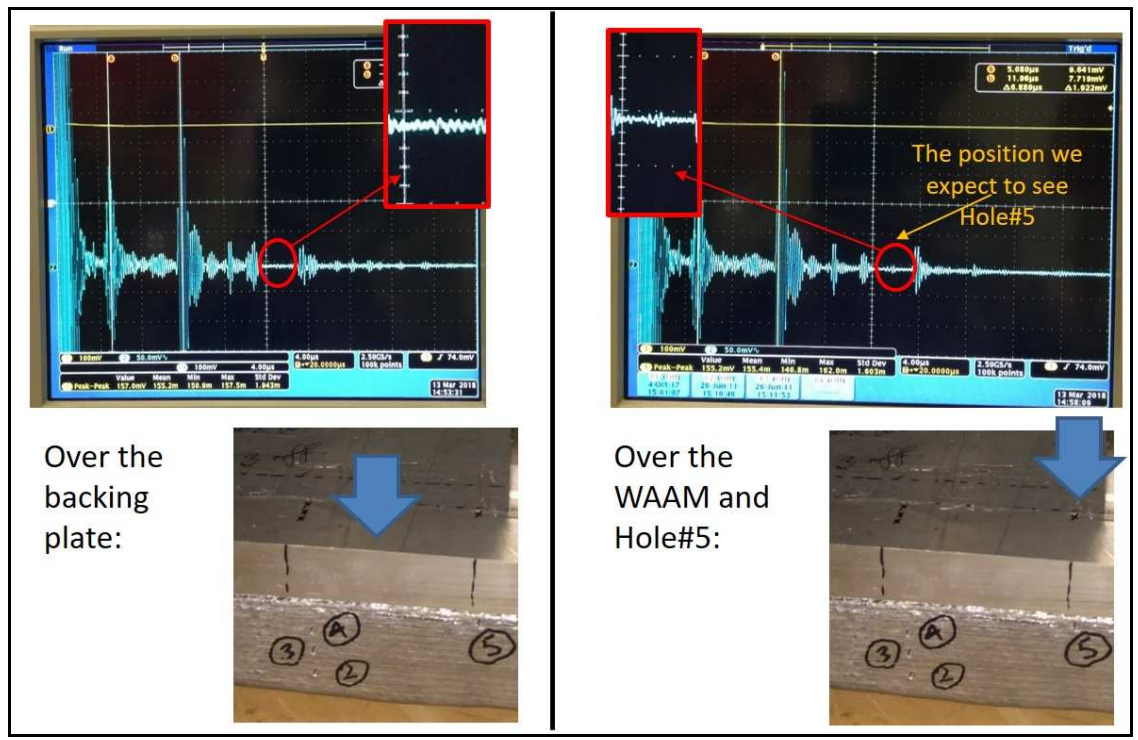

Figure 3. The difficulty of locating a $3 \mathrm{~mm}$ reflector by the single element ultrasonic prob 


\section{Ultrasonic phased array}

\subsection{As-built WAAM samples (ALT1 \& ALT2)}

\subsubsection{Conventional phased array}

ALT1 and ALT2 were inspected using a $5 \mathrm{MHz}$ ultrasonic array in direct contact with the backing plate surface using the conventional phased array method. A FIToolbox (Diagnostic Sonar, UK) was employed as the phased array controller. The B-scan performed was suitable to determine a majority of the reflectors and in the correct position, see Figure 4, however, the reflectors appear in an arc-shaped topology rather than a hole. Furthermore, the holes located near the surface were not detectable. These issues justified an application of total focusing method (TFM).

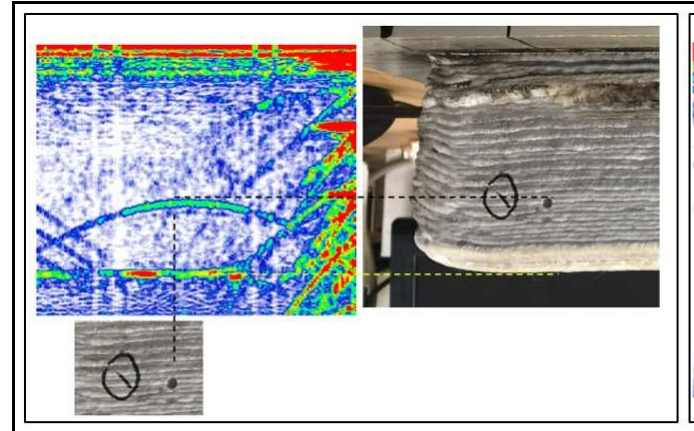

(a)

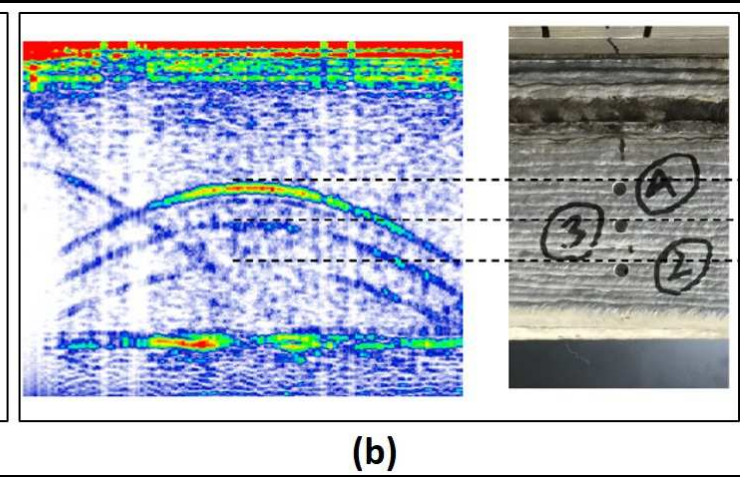

(b)

Figure 4. Conventional phased array scanning on ALT1

\subsubsection{Total focusing method (TFM)}

TFM was employed on ALT1 and AlT2 in order to determine all the defects and improve the defect shape in the scanning images, as is demonstrated in Figure 5. Using TFM, it was more practical to roughly measure the defect size (Figure 5a) as the hole-shaped defect topology is more realistic than what scanned by the conventional phased array (Figure 5b). Furthermore, the images created using TFM provided higher resolutions in comparison with those obtained from the conventional phased array (Figure 5c). Finally, the defects placed near the WAAM top surface were not detectable using the conventional method, while the TFM images provided a clear reproduction thereof (see Hole\#1 in Figure 5d). 


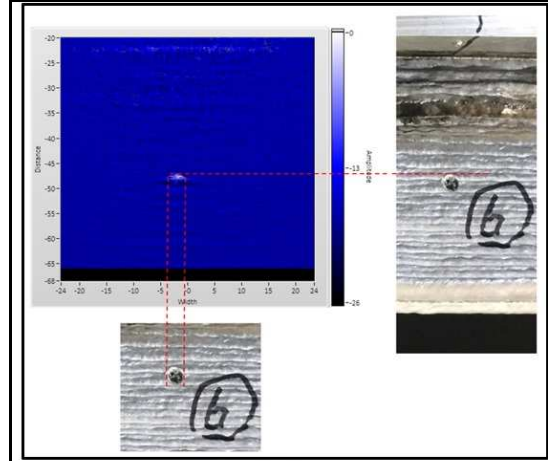

(a)

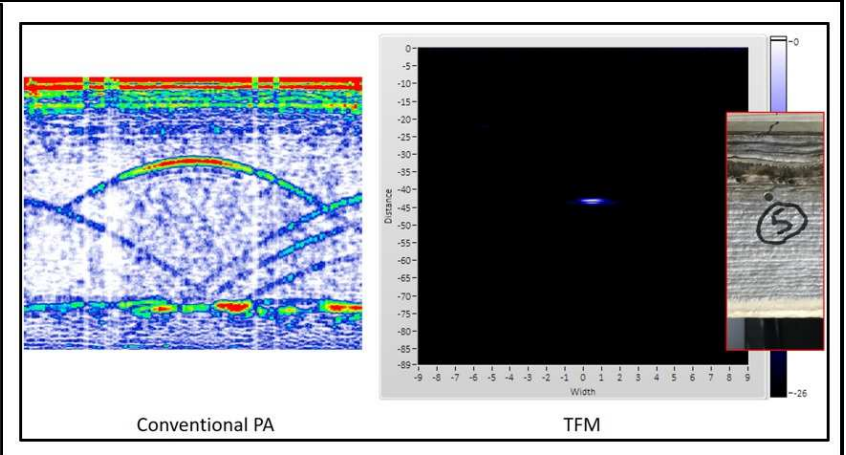

(b)

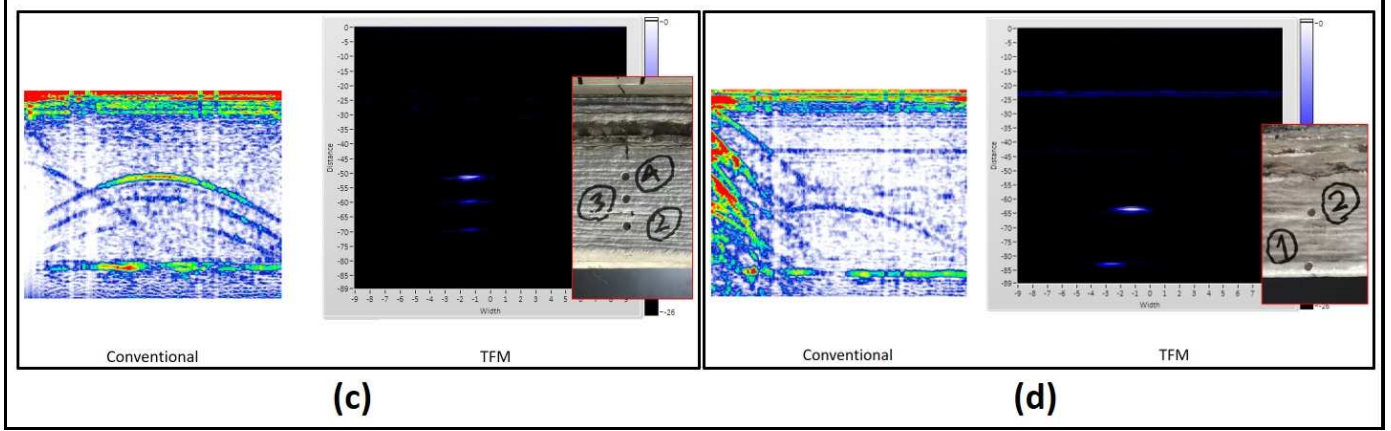

Figure 5. Comparison between the conventional phased array and TFM performed on ALT1 (a, b and c) and ALT2 (d)

\subsection{Machined WAAM sample (ALT3)}

\subsubsection{Conventional phased array using an improved aperture}

It is worth mentioning that only contact method is considered in this study because an application of the immersion method is impractical in the in-process WAAM-NDT which is the final goal of a larger project (RoboWAAM) supporting this sub-project work. Therefore, the WAAM top surface of ALT3 was machined to better facilitate ultrasonic scanning, providing a planar surface, rather than the complex, as-built surfaces of ALT1 and ALT2. Hence, ALT3 could be scanned by placing the array over either top surface or bottom surface (backing plate) while ALT1 and ALT2 were scanned only from the bottom surface. However, the artificial defects in the ALT3 were smaller than those made in the ALT1\&2 (see Table 1) and the majority of them were placed very close to the WAAM top surface. Hence, the phased array setting required to be improved in order to scan the ALT3. Some hardware modifications were carried out on the phased array system to allow exciting/receiving 32 elements simultaneously. The phased array controller allows performing a focussed B-scan with a range of settings for the transmit focal depth. Using these two improvements has resulted in detection of a majority of the artificial defects in the ALT3 scanned from the top 
surface, as shown in Figure 6. However, Figure 7 shows the difficulty of locating the nearsurface defects, even with a varied focal depth, due to the dead zone.

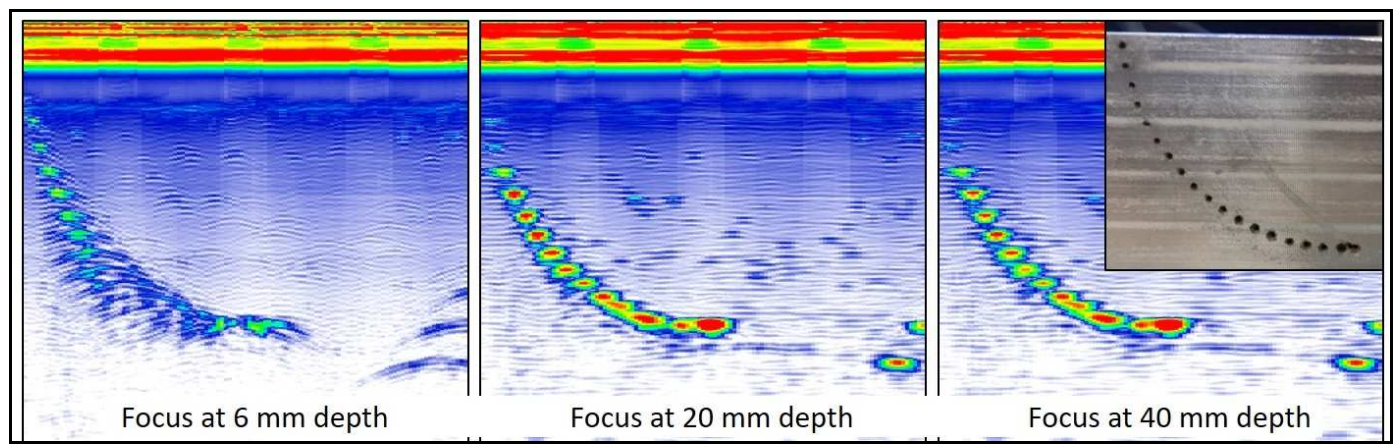

Figure 6. Focused B-Scan on ALT3

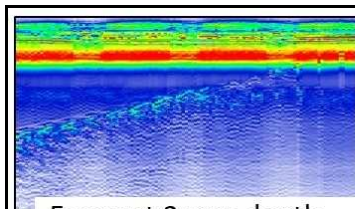

Focus at $2 \mathrm{~mm}$ depth

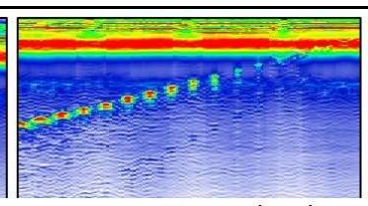

Focus at $5 \mathrm{~mm}$ depth

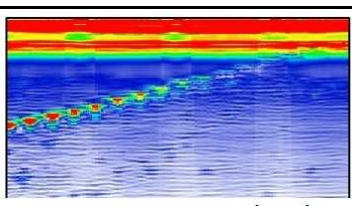

Focus at $20 \mathrm{~mm}$ depth

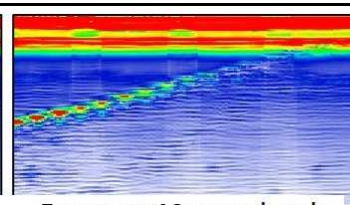

Focus at $40 \mathrm{~mm}$ depth
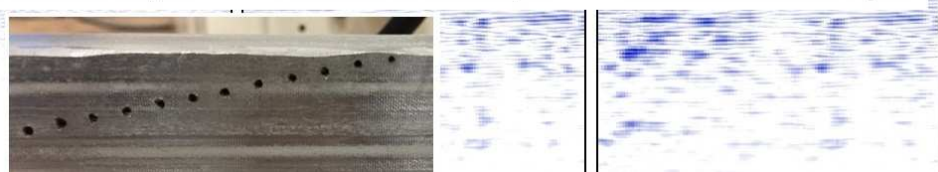

Figure 7. The difficulty of locating the near-surface defects in the focused B-Scan on ALT3

One solution for the near-surface defects is the application of a Perspex Wedge in order to remove the dead zone, Figure 8 . This solution was effective to detect a few more locators but not effective enough for detection of all the near surface defects.

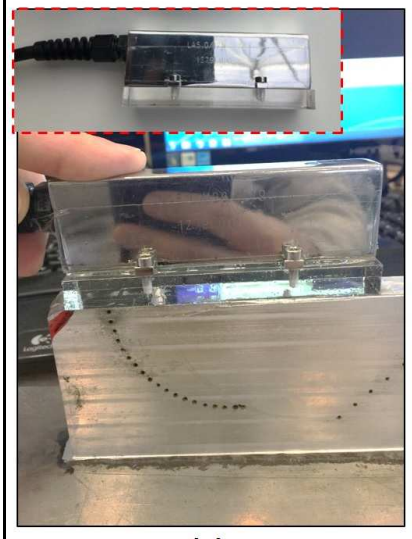

(a)

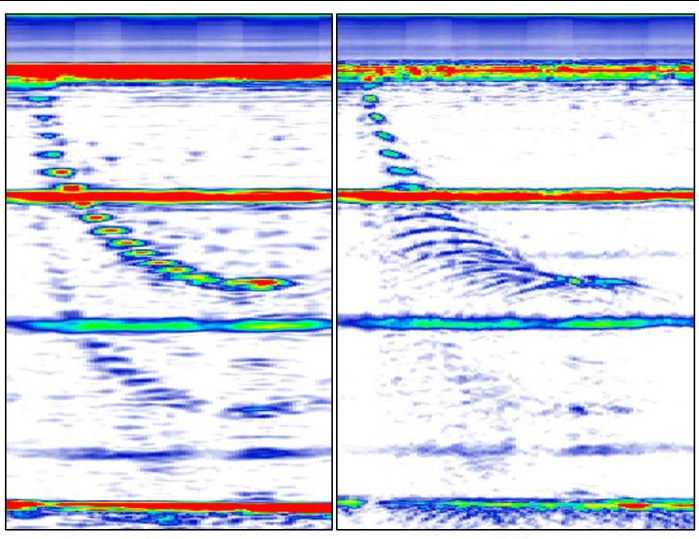

(b)

(c)

Figure 8. Using Perspex Wedge to remove the dead zone and resolve the problem of near-surface scanning on the ALT3 (a: setup, b: focus at $40 \mathrm{~mm}$ depth and c: focus at $10 \mathrm{~mm}$ depth) 
The second solution for the near-surface defects was the application of bottom surface scanning, putting the transducer over the backing plate. However, this resulted in some of the reflectors being missed due to the long distance the wave needed to travel in order to reach these specific holes, as evidenced in Figure 9. The holes which were close to the WAAM side wall were also not detected properly due to the side effects. These issues acted as the justification for using the TFM.

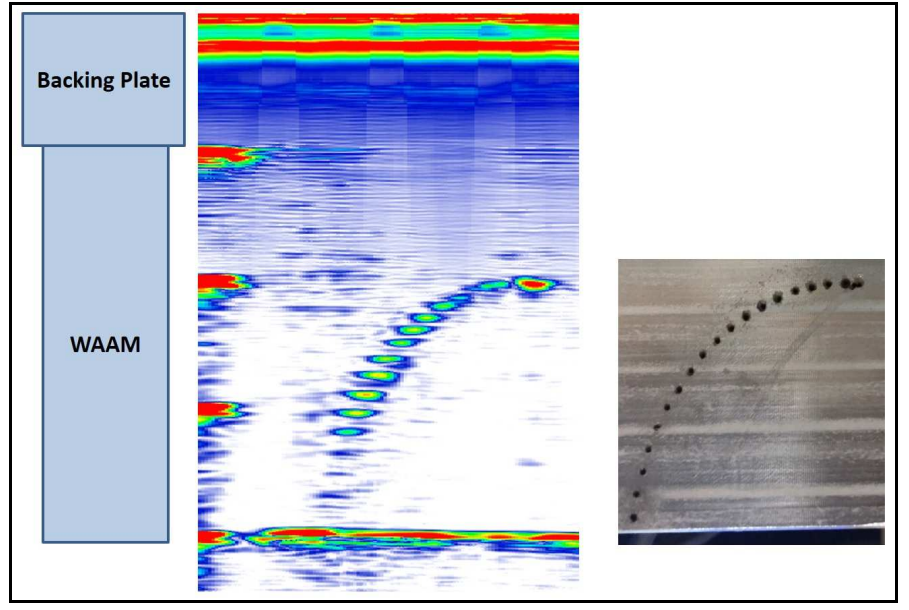

Figure 9. Bottom surface scanning of ALT3

\subsubsection{TFM on the ALT3}

The defects located near the WAAM sidewall were successfully detected using the TFM, as can be seen in Figure 10 .This contrasted with conventional phased array scanning, where the sidewall effect was a major issue. Regarding the near surface defects, TFM on the bottom surface improved the detectability of the defects considerably, as shown in Figure 11.

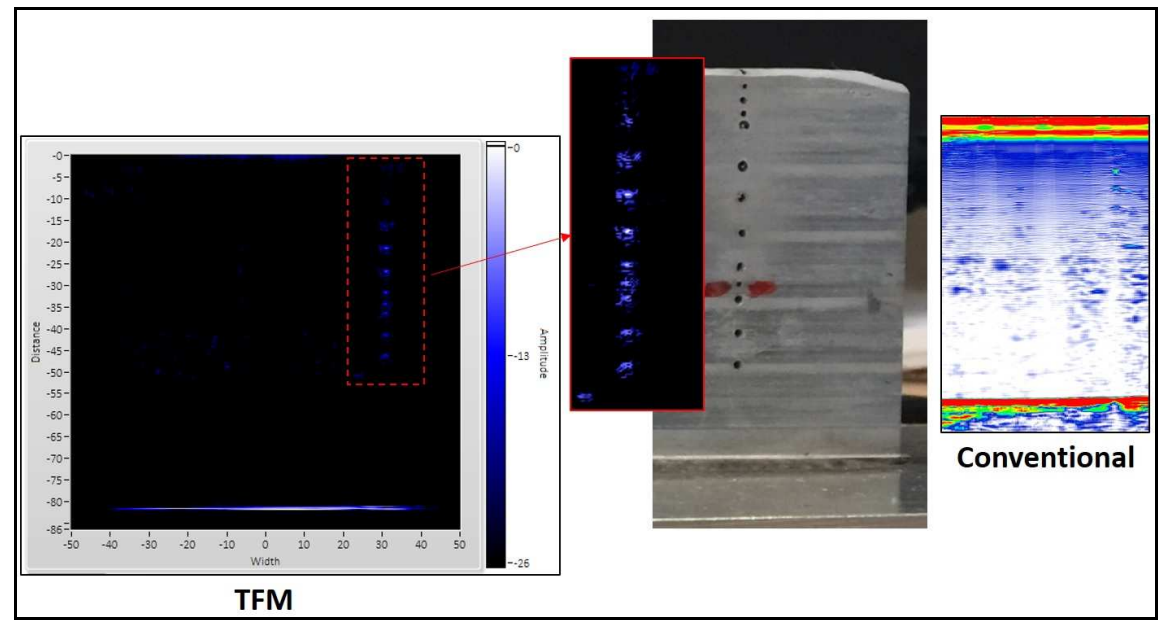

Figure 10. Resolving the side effect issue by using the TFM 


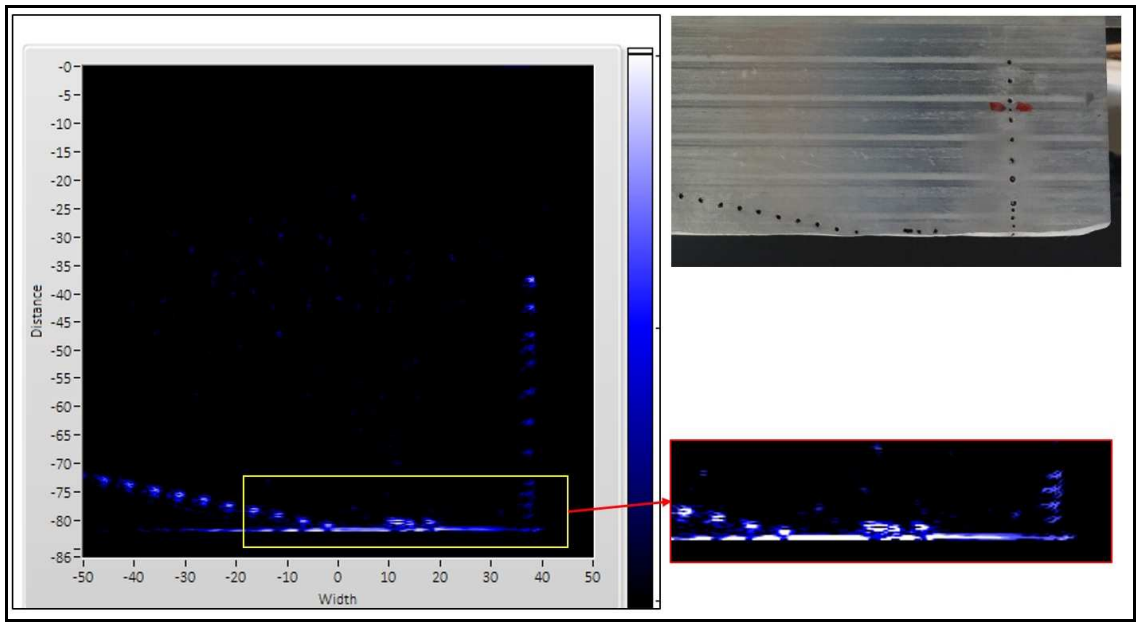

Figure 11. Inspection of the near surface defects using the TFM scanning over the backing plate

\subsubsection{Using $10 \mathrm{MHz}$ transducer}

While bottom surface scanning was capable of successfully detecting the near surface defects, top surface scanning is preferred in the larger research project (RoboWAAM) supporting this study. The phased array scanning techniques described so far were performed using a $5 \mathrm{MHz}$ array (128 elements). However, in this section, a $10 \mathrm{MHz}$ array (128 elements) was tested because it is a smaller transducer and has a lower dead zone. The smaller dead zone resulted in the reconstruction of almost all the near surface defects, although fairly masked in the dead zone, as shown in Figure 12. Furthermore, the $10 \mathrm{MHZ}$ array was found to produce clearer images in comparison with the $5 \mathrm{MHz}$ array, as seen in Figure 13.

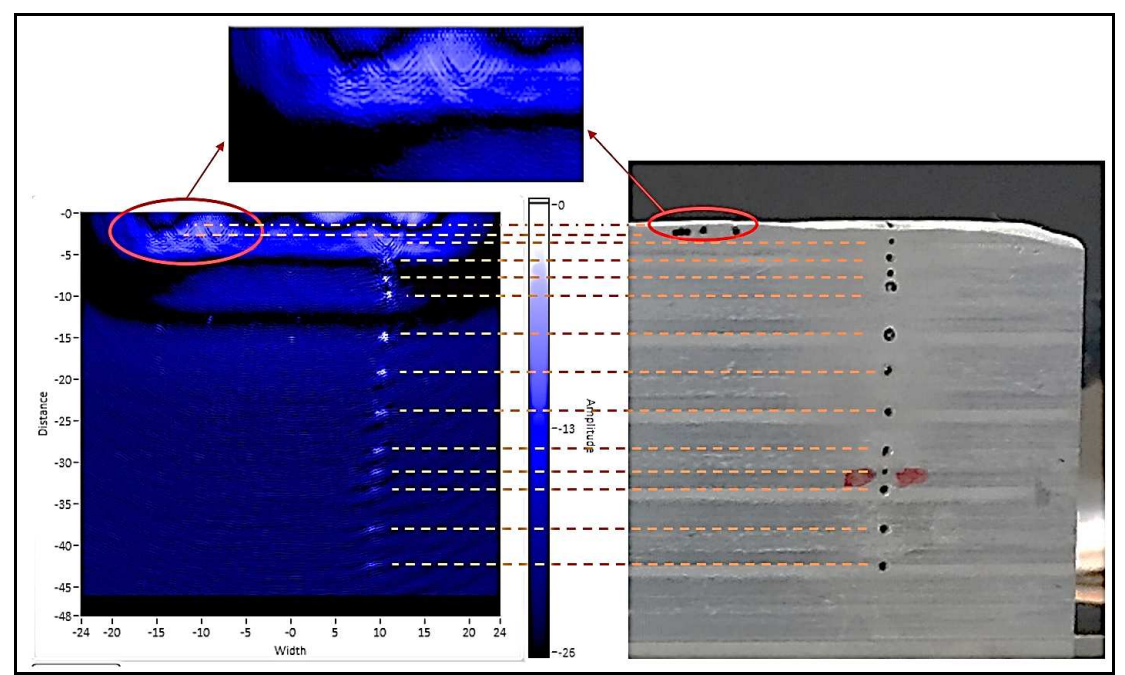

Figure 12. Near-surface defects scanned by the 10 MHz array placed over the WAAM top surface 


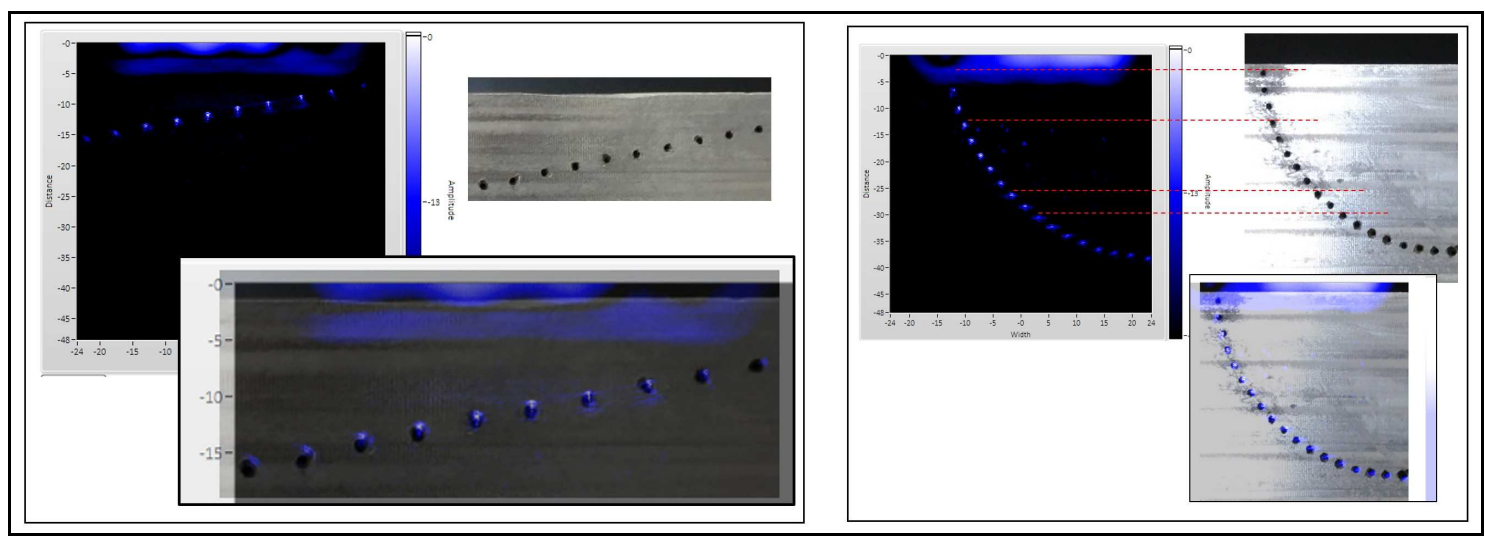

Figure 13. Improved resolution by using the $10 \mathrm{MHz}$ array for the TFM scanning of the ALT3

\section{Conclusions}

In this study, both conventional and TFM phased array methods were employed to inspect the WAAM aluminium samples. Based on the achieved results, it can be concluded that:

1) The majority of the defects in the WAAM sample were not detectable using a single element wheel probe. This resulted in the employment of ultrasonic phased array methods.

2) Conventional phased array methods provided a non-realistic defect shape (arc-shaped topology is shown instead of the hole) in the as-built samples. Furthermore, the defects near the WAAM top surface were not detectable using the conventional method. This was a justification for an application of TFM.

3) All the $ø 3 \mathrm{~mm}$ reflectors in the as-built samples, including the near-surface defects, were successfully detected by the TFM.

4) Regarding the $\varnothing 0.5 \mathrm{~mm}$ reflectors in the machined sample, hardware and software improvements in the conventional phased array were required. However, a combination of both conventional and TFM approaches were employed to detect all the reflectors including the near-surface defects.

5) Scanning from the WAAM bottom surface was found to be effective in the inspection of near-surface reflectors which are adjacent to the WAAM top surface.

6) Application of Perspex Wedge is also recommended to resolve the problem of the dead zone in the inspection. In this case, the application of the $10 \mathrm{MHz}$ array was also found to improve the scanning resolution. 
In total, all the reflectors in the WAAM specimens were successfully detected by a combination of conventional phased array and TFM along with a range of settings and setups including bottom surface inspection, perspex wedge application, changing the scanning frequency as well as an improved focused and varied aperture.

\section{Acknowledgements}

This work is supported by an EPSRC project (EP/P031064/1) called Robotic Wire + Arc Additive Manufacture (RoboWAAM). The authors then like to acknowledge EPSRC for the support and funding the project.

\section{References}

[1] J. Coykendall, M. Cotteleer, J. Holdowsky, M. Mahto, 3D opportunity in aerospace and defense: additive manufacturing takes flight, A Deloitte series on additive manufacturing, Deloitte University Press, Westlake, TX, 2014.

[2] C. Vargel, Corrosion of Aluminium, Elsevier Ltd., Oxford, 2004.

[3] S.W. Williams, F. Martina, A.C. Addison, J. Ding, G. Pardal, P. Colegrove, Wire plus Arc Additive Manufacturing, Materials Science and Technology 32 (2016) 641-647.

[4] B.W. Drinkwater, P.D. Wilcox, Ultrasonic arrays for non-destructive evaluation: A review, Ndt \& E International 39 (2006) 525-541.

[5] P. Cawley, Non-destructive testing - current capabilities and future directions, Proc. Inst. Mech. Eng. Pt. L-J. Mater.-Design Appl. 215 (2001) 213-223.

[6] Y. Mendelsohn, E. Wiener-Avnear, Simulations of circular 2D phase-array ultrasonic imaging transducers, Ultrasonics 39 (2002) 657-666.

[7] K.J. Kirk, A. McNab, A. Cochran, I. Hall, G. Hayward, Ultrasonic arrays for monitoring cracks in an industrial plant at high temperatures, Ieee Transactions on Ultrasonics Ferroelectrics and Frequency Control 46 (1999) 311-319.

[8] S. Chatillon, G. Cattiaux, M. Serre, O. Roy, Ultrasonic non-destructive testing of pieces of complex geometry with a flexible phased array transducer, Ultrasonics 38 (2000) 131-134.

[9] A. Neild, D.A. Hutchins, T.J. Robertson, L.A.J. Davis, D.R. Billson, The radiated fields of focussing aircoupled ultrasonic phased arrays, Ultrasonics 43 (2005) 183-195.

[10] A. Gachagan, S. Kelly, M. Altman, E. Grauvogl, G. Hayward, R. Banks, T. McCunnie, D. Skillen, Development of a manual air-coupled ultrasonic inspection instrument for use on aeronautical structures under in-service conditions, Review of Progress in Quantitative Nondestructive Evaluation, Vols 22a and $22 \mathrm{~b} 20$ (2003) 883-890.

[11] C. Holmes, B.W. Drinkwater, P.D. Wilcox, Post-processing of the full matrix of ultrasonic transmit-receive array data for non-destructive evaluation, Ndt \& E International 38 (2005) 701-711.

[12] C. Holmes, B.W. Drinkwater, P.D. Wilcox, Advanced post-processing for scanned ultrasonic arrays: Application to defect detection and classification in non-destructive evaluation, Ultrasonics 48 (2008) 636-642. [13] C.-P. Bazin de Caix, A. Gigant, ISO/TC 261: Additive manufacturing, in: B. Dutton (Ed.) NDT for AM parts, International Organization for Standardization, 2018.

[14] D. Clark, S.D. Sharples, D.C. Wright, Development of online inspection for additive manufacturing products, Insight 53 (2011) 610-613.

[15] A. Lopez, R. Bacelar, I. Pires, T. Santos, L. Quintino, MAPPING OF NON-DESTRUCTIVE TECHNIQUES FOR INSPECTION OF WIRE AND ARC ADDITIVE MANUFACTURING, Proceedings of the 7th International Conference on Mechanics and Materials in Design (M2d2017) (2017) 1829-1844.

[16] A. Lopez, R. Bacelar, I. Pires, T.G. Santos, J.P. Sousa, L. Quintino, Non-destructive testing application of radiography and ultrasound for wire and arc additive manufacturing, Additive Manufacturing 21 (2018) 298306. 